\title{
Improved Glycemic Control Through the Use of a Telehomecare Program in Patients with Diabetes Treated with Insulin
}

\author{
Annie Lemelin, MD, Ariane Godbout, MD, FRCPC, Guy Paré, $\mathrm{PhD}^{2}$ and Sophie Bernard, MD, $\mathrm{PhD}^{1,3}$
}

\begin{abstract}
Background: With the drastic surge in the prevalence of diabetes, the use of medical resources for management of diabetic patients increased markedly. This study aimed to evaluate the impact of telehomecare (THC) use on clinical efficacy, nursing interventions, and medical visits compared with the standard care in insulin-treated diabetic patients.

Materials and Methods: A prospective noninferiority clinical trial was designed. Participants were assigned to either an intervention group provided with a THC system during 3 months or to a control group. Main outcome was the difference in A1c at 3 months compared with baseline. Secondary outcomes were the difference in A1c at 6 months compared with baseline, the number of medical visits during the 6-month period of the study, and nursing interventions during the 3 months on THC.

Results: A total of 92 participants completed the study. A significant decrease in A1c levels was observed in the THC group $(n=45)$ compared with the control group $(n=47)$ at 3 months $(-0.61 \%$ vs. $-0.06 \%$, respectively, $P=0.048)$ and at 6 months $(-0.37 \%$ vs. $-0.10 \%$, respectively, $P=0.036)$. The THC group had an average of 0.6 medical visit compared with 1.0 in the control group $(P<0.001)$. An increase in nursing interventions (mainly e-mails) was noted in THC group ( $n=14.7)$ compared with control group $(n=1.1)$.

Conclusions: This THC program demonstrates improvement in glycemic control and a decrease in the number of medical visits. However, it is important to consider an additional burden in nursing interventions when implementing a THC program.
\end{abstract}

Keywords: Diabetes, Insulin, Telehomecare, Telemedicine, eHealth.

\section{Introduction}

$\mathbf{P}$ REVALENCE OF DIABETES has drastically increased in the past decade. In 2012, diabetes caused 1.5 million deaths worldwide. ${ }^{1}$ Diabetic patients require long-term follow-up to optimize diabetes control and prevent both macrovascular and microvascular complications. Indeed, a clear correlation between elevated A1c and diabetes-associated morbidity and mortality has been established. ${ }^{1,2}$ In addition, each percentage reduction in A1c through intensive glycemic control can reduce microvascular complications by $25 \%-30 \%$ as shown in patients with type 1 (DCCT Study) and type 2 (UKPDS Trial) diabetes. ${ }^{3,4}$ Unfortunately, $>50 \%$ of North American patients with diabetes have difficulty controlling their blood glucose and consequently have an A1c above target. ${ }^{5}$ Given the chronic nature of this disease, the patient's involvement in the management of diabetes on a daily basis is essential to prevent complications. The daily routine is a challenging process, including a healthy diet, regular physical activity, self-monitoring of blood glucose, carbohydrate counting, and insulin dose adjustments. Guidelines recommend to give an adequate support to the patients to acquire the knowledge and skills to optimize the management of their diabetes. ${ }^{5-7}$ In the long term, this approach not only improves clinical outcomes, but also the patient's health status and quality of life. ${ }^{5}$ Indeed, several meta-analyses, including both type 1 and type

\footnotetext{
${ }^{1}$ Endocrinology Division, Medicine Department, Centre de Recherche du Centre Hospitalier de l'Université de Montréal (CRCHUM), Montreal, Quebec, Canada.

${ }^{2}$ Research Chair in Digital Health, HEC Montreal, Montreal, Canada.

${ }^{3}$ Current address: IRCM, Montreal Research Institute, Montreal, Quebec, Canada.
} 
2 diabetic patients, have shown that self-management education is associated with improvement of $\mathrm{A} 1 \mathrm{c}$ and a reduction of diabetes-related complications. ${ }^{8}$

In this context, use of medical resources for education and management of diabetic patients increased markedly. Health care systems are, therefore, facing an increasing demand for resources and there is an urgent need to optimize care to maintain access, equity, quality, and cost-effectiveness. Telehomecare (THC) is a remote mode of intervention through electronic data transmission for follow-up, education, prevention as well as clinical decision-making, and therapeutic adjustment with great potential for the diabetic population. ${ }^{9,10}$ Furthermore, current literature suggests that education delivered through Internet/virtual mode is effective for diabetes education and for improvement of glycemic control both in type 1 and type 2 diabetic populations. $8,11,12$ It is also useful for patients with difficult access to care as it allows access to education and support at home, and also avoids the costs of a medical visit. Current data suggest that adhering to THC programs can permit active patient participation in the care process and encourage empowerment. ${ }^{10,13}$ THC intervention has demonstrated its effectiveness and efficiency in the management of several chronic conditions such as diabetes, chronic heart failure, and chronic obstructive pulmonary disease, among others. ${ }^{10,13}$ However, studies evaluating the use of THC in a population of insulintreated diabetic patients are still rare and the results are heterogeneous and mostly dedicated to type 1 diabetic populations. $^{14-17}$

The purpose of this study is to evaluate, in a real-life clinical setting, the impact of a THC program on clinical efficacy, nursing interventions, and medical visits compared with standard care in insulin-treated diabetic patients regardless of diabetes type.

\section{Materials and Methods}

\section{Study population}

This study is a prospective noninferiority and controlled clinical trial. Participants were recruited from the diabetes center outpatient clinic of the Centre Hospitalier de l'Université de Montréal (CHUM). We recruited participants during clinical visits (medical visit or nursing visit followup). Afterward, participants in the THC group were scheduled for an appointment to receive the teaching related to the THC platform.

The inclusion criteria were at least 18 years of age and type 1 or type 2 diabetes treated with at least one injection of insulin per day (with or without other oral/injectable antidiabetic agents). Exclusion criteria were inability to use the technology, insufficient understanding of the French language (since the browser was only available in French), and inability to give written consent to participate.

Participants were enrolled between December 3, 2015 and May 23, 2017. The study groups were formed sequentially: the THC group was formed first by asking eligible diabetes patients if they wanted to be followed with the THC system instead of usual care. The control group was formed in a period in which the clinic was not recruiting new participant on the platform.

The CHUM Research Ethics Board approved the study and all participants provided us with informed written consent.
Participants were assigned to either an intervention group provided with a THC system during 3 months for transmission and online analysis of capillary glucose or to a control group who received standard care. Then, both groups had standard care for a next 3-month period. The total duration of the study was 6 months.

\section{Standard care}

Based upon clinical judgment as in a real-life clinical setting, standard care included visits with the endocrinologist and nurses every 3-6 months. In cases of uncontrolled diabetes before the next planned visit, the participants contacted the care provider and a follow-up was scheduled by phone call or in person visit with the nurse or the endocrinologist as required.

\section{THC system}

The THC system was developed with the help of Orion Health/Bell. Participants in the THC group either used a dedicated tablet provided by the health care team or accessed services through a web browser through their personal computer/tablet/phone. Participants used the THC system to register their daily health activities personalized by their care provider. All participants were asked to record their capillary blood glucose values fasting as well as before and/or $2 \mathrm{~h}$ after meals. Hypoglycemia alert was defined as a capillary blood glucose $<4.0 \mathrm{mmol} / \mathrm{L}$ and hyperglycemia alert as a capillary blood glucose $>15.0 \mathrm{mmol} / \mathrm{L}$. The participants were asked to register their capillary blood glucose values in the THC system on a daily basis. In certain circumstances, the participants may not be able to enter their capillary blood glucose values every day (especially during holidays), but it was required that during these periods they registered their capillary blood glucose values in the system at least once a week. The glycemic control assessment was done online daily by the nurse if there were any alerts (such as hypoglycemia or hyperglycemia) and there was a review online of all data and charts with the medical team at least every 2 weeks for all participants (Supplementary Figs. S1-S5 in Supplementary Data). When required, participants came to the outpatient clinic to receive specific intervention by the clinical team.

Coaching was delivered directly through the platform, repetitively and interactively when required, by the clinical team or automatically from a set of preprogrammed algorithms activated if the participant entered blood glucose values out of the normal range. Each time a result classified as hypo- or hyperglycemia was detected by the platform, a set of different questions and instructions for the participant regarding their symptoms, potential reasons for out-of-range values, their diet, physical activity, and, if applicable, insulin treatment was matched for each case asking afterward in most situation for a timely control of blood glucose.

Automated and adjusted feedback provided directly to the participant by those preprogrammed clinical algorithms promotes better health behaviors and help participants to adjust adequately their diet, lifestyle, or their insulin therapy.

Also, participants had access to a virtual library of diabetes information. Clinical data were securely transmitted, stored, and accessible to caregivers (nurses). Nursing interventions included e-mails, phone calls, and in person visits. 
The participants' THC files as well as the medical notes were added to the participants' electronic medical record.

\section{Objectives}

The primary outcome was the difference observed in A1c at 3 months compared with baseline between the THC group and the control group in insulin-treated diabetic patients.

Secondary objectives were the difference observed in A1c between the two groups at 6 months compared with baseline, the number of medical visits related to diabetes during the 6-month period of the study, and nursing interventions (number of in person visits, phone calls, and e-mails) during the 3 months on THC.

\section{Statistical analysis}

Collected data were analyzed at 3 and 6 months after the inclusion visit. Analyses were conducted with the IBM SPSS software version 25 .

Categorical data are expressed as frequencies and percentages, whereas quantitative data are expressed as means and standard deviations. The analysis of covariance (ANCOVA) was used to compare groups for primary endpoint results. ANCOVA, chi-square test, and Fisher exact test were used for other comparisons. Univariate linear regression analy- sis was performed to determine variables associated with change in A1c at 3 months compared with baseline in the THC group. A $P$ value $<0.05$ was considered to indicate statistical significance.

\section{Results}

A total of 105 participants were recruited: 54 in the THC group and 51 in the control group. Ten participants withdrew from the study (six in the THC group and four in the control group) (Fig. 1). Therefore, 45 participants in THC group (83\%) and 47 participants in the control group (92\%) completed the study. Main clinical and diabetes characteristics at inclusion are shown in Table 1. Participants in the THC group have significantly more diabetic nephropathy and the control group participants have more dyslipidemia and a longer duration of diabetes at baseline.

Compared with baseline, a significant decrease in A1c level was observed after 3 months of follow-up in the THC group with a reduction of $-0.61 \%$ compared with $-0.06 \%$ in the control group $(P=0.048)$. The significant decrease in A1c was maintained at 6 months in the THC group compared with the control group $(-0.37 \%$ vs. $-0.10 \%$, respectively, $P=0.036)$ (Table 2).

The THC group had an average of 0.6 medical visits related to the diabetes compared with 1.0 in the control group

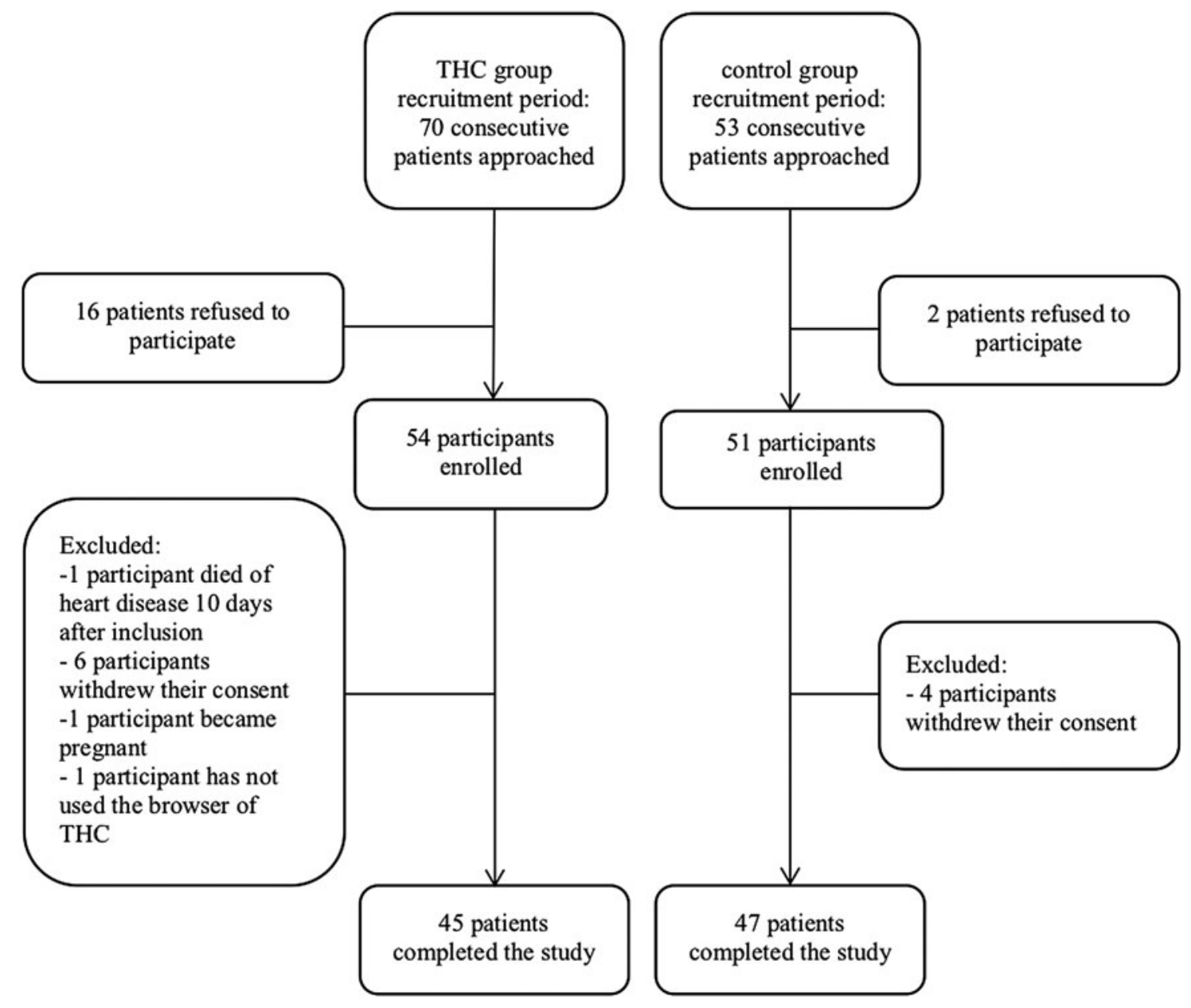

FIG. 1. CONSORT diagram. 
Table 1. Baseline Characteristics of Patients

\begin{tabular}{|c|c|c|c|}
\hline & THC group $(\mathrm{n}=45)$ & Control group $(\mathrm{n}=47)$ & $\mathrm{P}$ \\
\hline \multicolumn{4}{|l|}{ Gender $(\%)$} \\
\hline Men & 40.0 & 59.6 & 0.060 \\
\hline $\begin{array}{l}\text { Age (years) } \\
\text { BMI }\left(\mathrm{kg} / \mathrm{m}^{2}\right)\end{array}$ & $\begin{array}{c}59 \pm 13 \\
29.1 \pm 5.8\end{array}$ & $\begin{array}{c}57 \pm 15 \\
31.8 \pm 7.6\end{array}$ & $\begin{array}{l}0.629 \\
0.064\end{array}$ \\
\hline \multicolumn{4}{|l|}{ Type of diabetes (\%) } \\
\hline Type 2 & 55.6 & 42.6 & 0.114 \\
\hline Type 1 & 28.9 & 53.2 & \\
\hline LADA & 8.9 & 2.1 & \\
\hline Pancreatic & 4.4 & 2.1 & \\
\hline Corticosteroid induced & 2.2 & 0.0 & \\
\hline Duration of diabetes (years) & $19.1 \pm 12$ & $28.7 \pm 14$ & $<\mathbf{0 . 0 0 1}$ \\
\hline $\mathrm{A} 1 \mathrm{c}(\%)$ & 8.1 & 7.8 & 0.425 \\
\hline \multicolumn{4}{|l|}{ Associated comorbidities (\%) } \\
\hline Cardiovascular disease & 22.7 & 15.2 & 0.36 \\
\hline Peripheral vascular disease & 4.5 & 10.9 & 0.26 \\
\hline Stroke & 6.8 & 2.2 & 0.36 \\
\hline Hypertension & 61.4 & 69.6 & 0.41 \\
\hline Dyslipidemia & 65.9 & 87.0 & 0.02 \\
\hline Chronic kidney disease & 11.4 & 8.7 & 0.74 \\
\hline \multicolumn{4}{|l|}{ Microvascular complications (\%) } \\
\hline Retinopathy & 39.5 & 32.6 & 0.50 \\
\hline Nephropathy & 44.2 & 6.5 & $<\mathbf{0 . 0 0 1}$ \\
\hline Neuropathy & 38.1 & 56.5 & 0.08 \\
\hline Number of insulin injections per day & $4.0 \pm 0.8$ & $3.8 \pm 1.3$ & 0.207 \\
\hline Insulin $(\mathrm{U} / \mathrm{kg})$ & $0.85 \pm 0.46$ & $0.94 \pm 0.75$ & 0.523 \\
\hline
\end{tabular}

Values are expressed as mean \pm SD or percentage as indicated. Bold values indicate $P<0.05$.

LADA, latent autoimmune diabetes in adults; SD, standard deviation; THC, telehomecare.

during the THC monitoring period $(P<0.001)$ (Table 2$)$. An increase in nursing interventions was noted in the THC group $(n=14.7)$ compared with the control group $(n=1.5)$ (Table 2).

In the THC group, the adherence was good since the mean total number of capillary blood glucose monitoring during the 3 -month period was $289.66 \pm 106.99$. Only one participant recorded a total of 19 values in the system. E-mails were the most frequent interaction with participants in the THC group, representing $91.5 \%$ of the nursing interventions compared with $7.0 \%$ for phone calls and $1.5 \%$ for in person visits. The average time spent per participant by the caregiver, including e-mails and phone calls for the 3-month follow-up period was $328.97 \pm 131.18 \mathrm{~min}$.

In univariate analysis, the baseline A1c was the only parameter associated with the observed change in A1c at 3 months in the THC group (Table 3). Participants with a

Table 2. Diabetes Control and Medical Follow-Up

\begin{tabular}{|c|c|c|c|}
\hline & THC group $(\mathrm{n}=45)$ & Control group $(\mathrm{n}=47)$ & $\mathrm{P}$ \\
\hline \multicolumn{4}{|l|}{ Mean A1c (\%) } \\
\hline Baseline & $8.11 \pm 1.8$ & $7.83 \pm 1.6$ & 0.425 \\
\hline 3 months & $7.47 \pm 1.6$ & $7.69 \pm 1.4$ & 0.495 \\
\hline 6 months & $7.45 \pm 2.1$ & $7.65 \pm 1.2$ & 0.625 \\
\hline \multicolumn{4}{|l|}{ Mean difference in A1c } \\
\hline 3 months compared with baseline & $-0.61 \pm 1.3$ & $-0.06 \pm 1.1$ & 0.048 \\
\hline 6 months compared with baseline & $-0.37 \pm 1.2$ & $-0.10 \pm 0.5$ & $\mathbf{0 . 0 3 4}$ \\
\hline 6 months compared with 3 months & $-0.06 \pm 1.1$ & $0.00 \pm 0.7$ & 0.816 \\
\hline \multicolumn{4}{|l|}{ Medical visits $(n)$} \\
\hline 6-month period before inclusion & $1.7 \pm 1.2$ & $0.8 \pm 0.7$ & $<\mathbf{0 . 0 0 1}$ \\
\hline 3-month period on THC & $0.6 \pm 0.6$ & $1.0 \pm 0.4$ & $<0.001$ \\
\hline 3-month period after THC & $0.4 \pm 0.6$ & $0.7 \pm 0.6$ & 0.058 \\
\hline \multicolumn{4}{|c|}{ Nursing interventions during 3-month period on THC $(n)$} \\
\hline Follow-up at the clinic & $1.8 \pm 0.9$ & $1.0 \pm 0.2$ & $<\mathbf{0 . 0 0 1}$ \\
\hline Follow-up by phone & $4.8 \pm 2.7$ & $0.3 \pm 0.5$ & \\
\hline Follow-up by e-mail & $8.1 \pm 3.4$ & $0.2 \pm 0.4$ & \\
\hline Total & $14.7 \pm 5.0$ & $1.5 \pm 0.7$ & \\
\hline
\end{tabular}

Values are expressed as mean \pm SD. Bold values indicate $P<0.05$. 
Table 3. Univariate Analysis of Factors ASSOCIATED WITH VARIATION IN A1C at 3 Months In the Telehomecare Group

\begin{tabular}{lcc}
\hline Variable & $\begin{array}{c}\text { Standardized } \\
\text { beta-coefficient }\end{array}$ & $\mathrm{P}$ \\
\hline Gender & -0.040 & 0.81 \\
Duration of diabetes & 0.121 & 0.49 \\
Type of diabetes & -0.316 & 0.09 \\
Diabetes complications & -0.019 & 0.91 \\
$\begin{array}{l}\text { Baseline A1c } \\
\text { Number of glycemia entered } \\
\text { in the system }\end{array}$ & -0.516 & $\mathbf{0 . 0 0 1}$ \\
$\begin{array}{l}\text { Time required for caregiver } \\
\text { per participants }\end{array}$ & 0.010 & 0.95 \\
\hline
\end{tabular}

Bold value indicates $P<0.05$.

higher A1c at baseline had a greater decrease in A1c at the end of the 3-month period on THC.

The number of injections, total daily insulin dose, and antidiabetic medications were not different at 3 months compared with baseline in the THC group.

\section{Discussion}

Our study demonstrated a significant decrease in A1c in the THC group compared with the group with standard care, which persisted 3 months after the end of the THC use. The observed effect on glycemic control is consistent with the current literature on THC use in diabetes care. Two recent meta-analyses assessing telemedicine use in diabetes have shown a significant reduction in A1c compared with standard care: $-0.44 \%$ for all type of diabetes combined ${ }^{16}$ and $-0.54 \%$ for type 2 diabetes. ${ }^{18}$ It should be noted that the impact on glycemic control found in our study, with a reduction of $0.61 \%$ in A1c, is similar to that observed with some antidiabetic drugs. ${ }^{19}$ The improvement in glycemic control may be explained by the intensive glycemic monitoring and rapid therapeutic adjustments by the participants and care providers. However, the change in A1c observed at 3 months in the THC group was only associated with the baseline A1c and not with the number of glucose monitoring entered in the system nor the time of nursing interventions. The improvement in glucose control may also be related to better lifestyle, since the participant had to mention if the observed high blood glucose was related to higher intake of carbs or physical inactivity. Instructions delivered in real time to the participants by algorithms to correct hypoglycemia or hyperglycemia may also have contributed to the reduction of A1c. The main original feature of our system relied on these preprogrammed algorithms that have helped the participants to improve the diabetes self-management.

The decrease in Alc observed in our study was maintained in time even after stopping THC monitoring. These results suggest that participants using the THC platform developed empowerment, a key aspect in the self-management of diabetes. Our study also demonstrated a significant decrease in medical visits between the THC group and the standard care group without compromising quality of care. This is in agreement with a retrospective Canadian study, evaluating cost-effectiveness, which showed a significant reduction in endocrinologist visits for diabetic patients in the telemedicine group compared with the control group. ${ }^{20}$ Furthermore, a recent study published by Davidson also reported a pilot project using a remote glucose monitoring utilizing computerized insulin dose adjustment and results showed that the method was safe and effective while saving medical time and face-to-face visit. ${ }^{21}$ However, it should be noted that our study reflected a short-term analysis for a 6-month period and that long-term impact on medical visits associated with THC monitoring remains to be explored. Moreover, despite a reduction in medical visits, an additional burden in nursing interventions is expected when implementing a THC system and should be taken into consideration.

One of the major limitations of our study is the absence of formal randomization despite a sequential buildup of the cohorts to limit recruitment bias. Another limitation of the study is due to missing information regarding hypoglycemia and hyperglycemia at baseline. Finally, no cost-benefit analyses of THCs were conducted. A recent systematic review reports that telemedicine has the potential to reduce the cost of care in diabetes management, but only a few studies have evaluated the cost-effectiveness. ${ }^{22}$

In conclusion, this study suggests the usefulness of THC interventions in diabetes care with improvements in glycemic control associated with a decreased number of medical visits. It suggests that THC use for the care of diabetic patients treated with insulin appears both safe and effective. These results open up the possibility of multiple uses for THCs in the care of diabetic patients, especially in patients with poorly controlled diabetes, during treatments with a hyperglycemic drug, or for vulnerable populations with limited access to care. Further trials are needed to provide specific cost-effectiveness assessments of THC interventions to allow substantial deployment and health care providers' support.

\section{Acknowledgments}

The THC system was developed with the help of Orion Health/Bell. The authors thank Nathalie Saint-Pierre (research coordinator at Institut de Recherches Cliniques de Montréal) for her helpful proofreading and feedback, Martine Paquette (research coordinator at Institut de Recherches Cliniques de Montréal) for the additional statistical analysis, as well as all participants and medical staff involved.

\section{Author Disclosure Statement}

No competing financial interests exist.

\section{Funding Information}

Canada Health Infoway, Orion Health, Bell and the Quebec Health care authorities provided funding and support for the study. No employees from these organizations were involved in data collection and analysis, or in the writing of this report.

\section{Supplementary Material}

Supplementary Figure S1 Supplementary Figure S2 Supplementary Figure S3 Supplementary Figure S4 Supplementary Figure S5 


\section{References}

1. Klein R, Klein BE, Moss SE, et al.: Glycosylated hemoglobin predicts the incidence and progression of diabetic retinopathy. JAMA 1988;260:2864-2871.

2. Stratton IM, Adler AI, Neil HA, et al.: On behalf of the UK Prospective Diabetes Study Group: Association of glycaemia with macrovascular and microvascular complications of type 2 diabetes (UKPDS 35): prospective observational study. BMJ 2000;321:405-412.

3. Nathan DM, Genuth S, Lachin J, et al.: The effect of intensive treatment of diabetes on the development and progression of long-term complications in insulin-dependent diabetes mellitus. N Engl J Med 1993;329:977-986.

4. UKPDS: Intensive blood-glucose control with sulphonylureas or insulin compared with conventional treatment and risk of complications in patients with type 2 diabetes (UKPDS 33). UK Prospective Diabetes Study (UKPDS) Group. Lancet 1998;352:837-853.

5. Canadian Diabetes Association Clinical Practice Guidelines Expert Committee, Cheng AY: Canadian Diabetes Association 2013 Clinical Practice Guidelines for the Prevention and Management of Diabetes in Canada. Introduction. Can J Diabetes 2013;37 Suppl 1:S1-S3.

6. Bodenheimer T, Lorig K, Holman H, et al.: Patient selfmanagement of chronic disease in primary care. JAMA 2002;288:2469-2475.

7. Funnell MM, Anderson RM: Empowerment and selfmanagement of diabetes. Clin Diabetes 2004;22:123-127.

8. Worswick J, Wayne SC, Bennett R, et al.: Improving quality of care for persons with diabetes: an overview of systematic reviews-what does the evidence tell us? Syst Rev 2013;2:26.

9. Pare G, Jaana M, Sicotte C: Systematic review of home telemonitoring for chronic diseases: the evidence base. J Am Med Inform Assoc 2007;14:269-277.

10. Wildevuur SE, Simonse LW: Information and communication technology-enabled person-centered care for the "big five" chronic conditions: scoping review. J Med Internet Res 2015;17:e77.

11. Pereira K, Phillips B, Johnson C, et al.: Internet delivered diabetes self-management education: a review. Diabetes Technol Ther 2015;17:55-63.

12. Cotter AP, Durant N, Agne AA, et al.: Internet interventions to support lifestyle modification for diabetes management: a systematic review of the evidence. J Diabetes Complications 2014;28:243-251.

13. Biermann E, Dietrich W, Rihl J, et al.: Are there time and cost savings by using telemanagement for patients on intensified insulin therapy? A randomised, controlled trial. Comput Methods Programs Biomed 2002;69:137-146.
14. Montori VM, Helgemoe PK, Guyatt GH, et al.: Telecare for patients with type 1 diabetes and inadequate glycemic control: a randomized controlled trial and meta-analysis. Diabetes Care 2004;27:1088-1094.

15. Benhamou PY, Melki V, Boizel R, et al.: One-year efficacy and safety of Web-based follow-up using cellular phone in type 1 diabetic patients under insulin pump therapy: the PumpNet study. Diabetes Metab 2007;33:220-226.

16. Rossi MC, Nicolucci A, Di Bartolo P, et al.: Diabetes Interactive Diary: a new telemedicine system enabling flexible diet and insulin therapy while improving quality of life: an open-label, international, multicenter, randomized study. Diabetes Care 2010;33:109-115.

17. Charpentier G, Benhamou PY, Dardari D, et al.: On behalf of the TeleDiab Study Group: The Diabeo software enabling individualized insulin dose adjustments combined with telemedicine support improves $\mathrm{HbAlc}$ in poorly controlled type 1 diabetic patients: a 6-month, randomized, open-label, parallel-group, multicenter trial (TeleDiab 1 Study). Diabetes Care 2011;34:533-539.

18. Huang Z, Tao H, Meng Q, et al.: Management of endocrine disease. Effects of telecare intervention on glycemic control in type 2 diabetes: a systematic review and metaanalysis of randomized controlled trials. Eur J Endocrinol 2015;172:R93-R101.

19. Committee DCCPGE: Diabetes Canada 2018 Clinical Practice Guidelines for the Prevention and Management of Diabetes in Canada. Can J Diabetes 2018;42(Suppl 1):S1-S325.

20. Deng L, White AS, Pawlowska M, et al.: Cost-benefit analysis of internet therapeutic intervention on patients with diabetes. Int J Endocrinol Metab 2015;13:e22803.

21. Davidson MB, Davidson SJ: Effect of remote glucose monitoring utilizing computerized insulin dose adjustment algorithms: a pilot project. Diabetes Ther 2019;10:523-533.

22. Lee JY, Lee SWH: Telemedicine cost-effectiveness for diabetes management: a systematic review. Diabetes Technol Ther 2018;20:492-500.

Address correspondence to:

Sophie Bernard, MD, PhD

Institut de recherches cliniques de Montréal (IRCM)

Montreal Clinical Research Institute 110, avenue des Pins Ouest Montréal H2W $1 R 7$ Quebec, Canada

E-mail: sophie.bernard@ircm.qc.ca 